\title{
The spectrum of discrete Dirac operator with a general boundary condition
}

\author{
Nimet Coskun ${ }^{1 *}$ (i) and Nihal Yokus ${ }^{1}$
}

\author{
"Correspondence: \\ cannimet@kmu.edu.tr \\ ${ }^{1}$ Department of Mathematics, \\ Karamanoglu Mehmetbey \\ Univercity, Yunus Emre Yerleşkesi, \\ 70100, Karaman, Turkey
}

\begin{abstract}
In this paper, we aim to investigate the spectrum of the nonselfadjoint operator $L$ generated in the Hilbert space $I_{2}\left(\mathbb{N}, \mathbb{C}^{2}\right)$ by the discrete Dirac system

$$
\left\{\begin{array}{l}
y_{n+1}^{(2)}-y_{n}^{(2)}+p_{n} y_{n}^{(1)}=\lambda y_{n}^{(1)}, \\
-y_{n}^{(1)}+y_{n-1}^{(1)}+q_{n} y_{n}^{(2)}=\lambda y_{n}^{(2)},
\end{array} \quad n \in \mathbb{N},\right.
$$

and the general boundary condition

$$
\sum_{n=0}^{\infty} h_{n} y_{n}=0
$$

where $\lambda$ is a spectral parameter, $\Delta$ is the forward difference operator, $\left(h_{n}\right)$ is a complex vector sequence such that $h_{n}=\left(h_{n}^{(1)}, h_{n}^{(2)}\right)$, where $h_{n}^{(i)} \in I^{1}(\mathbb{N}) \cap R^{2}(\mathbb{N}), i=1,2$, and $h_{0}^{(1)} \neq 0$. Upon determining the sets of eigenvalues and spectral singularities of $L$, we prove that, under certain conditions, $L$ has a finite number of eigenvalues and spectral singularities with finite multiplicity.
\end{abstract}

MSC: 39A10; 39A12; 47A10; 47A75

Keywords: Eigenparameter; Spectral analysis; Eigenvalues; Spectral singularities; Discrete equation; Dirac equation

\section{Introduction}

Along with the invention of the Schrödinger equation, the physical scope of mathematical problems connected with the spectra of differential equations with prescribed boundary conditions was enormously enlarged. The types of equations that previously had applications only to mechanical vibrations now were to be used for the description of atoms and molecules. There are important and altogether astonishing applications of the results obtained in the spectral theory of linear operators in Hilbert spaces to scattering theory, inverse problems, and quantum mechanics. For instance, the Hamiltonian of a quantum particle confined to a box involves a choice of boundary conditions at the box ends. Since different choices of boundary conditions imply different physical models, spectral theory of operators with boundary conditions constitues a progressing field of investigation $[1,2]$.

(c) The Author(s) 2020. This article is licensed under a Creative Commons Attribution 4.0 International License, which permits use, sharing, adaptation, distribution and reproduction in any medium or format, as long as you give appropriate credit to the original author(s) and the source, provide a link to the Creative Commons licence, and indicate if changes were made. The images or other third party material in this article are included in the article's Creative Commons licence, unless indicated otherwise in a credit line to the material. If material is not included in the article's Creative Commons licence and your intended use is not permitted by statutory regulation or exceeds the permitted use, you will need to obtain permission directly from the copyright holder. To view a copy of this licence, visit http://creativecommons.org/licenses/by/4.0/. 
Let $T$ denote a matrix operator

$$
T=\left(\begin{array}{ll}
p_{11}(x) & p_{12}(x) \\
p_{21}(x) & p_{22}(x)
\end{array}\right), \quad p_{12}(x)=p_{21}(x),
$$

where $p_{i k}(x)(i, k=1,2)$ are real continuous functions on the interval [0, $\left.\pi\right]$. Let also $y(x)$ denote a two-component vector function

$$
y(x)=\left(\begin{array}{l}
y_{1}(x) \\
y_{2}(x)
\end{array}\right) .
$$

If

$$
B=\left(\begin{array}{cc}
0 & 1 \\
-1 & 0
\end{array}\right), \quad I=\left(\begin{array}{ll}
1 & 0 \\
0 & 1
\end{array}\right),
$$

and $\lambda$ is a parameter, then the equation

$$
\left(B \frac{d}{d x}+T-\lambda I\right) y=0
$$

is equivalent to a system of two simultaneous first-order ordinary differential equations

$$
\begin{aligned}
& \frac{d y_{2}}{d x}+p_{11}(x) y_{1}+p_{12}(x) y_{2}=\lambda y_{1}, \\
& -\frac{d y_{1}}{d x}+p_{21}(x) y_{1}+p_{22}(x) y_{2}=\lambda y_{2} .
\end{aligned}
$$

In the case of $p_{12}(x)=p_{21}(x)=0, p_{11}(x)=V(x)+m$, and $p_{22}(x)=V(x)-m$, where $V(x)$ is a potential function, and $m$ is the mass of a particle, system (1.1) is called a stationary one-dimensional Dirac system in relativistic quantum theory. Levitan and Sargsjan [1] have introduced some basic concepts regarding the general spectral theory of self-adjoint Sturm-Liouville and Dirac operators and presented a discrete analogue of system (1.1) using the method of finite differences. If the functions $p_{i k}(x)(i, k=1,2)$ are complex valued, then the operator $T$ is called nonselfadjoint. Also, if the operator $T$ is defined on an infinite interval, then it is said to be singular. The structure of the spectrum of the operator $T$ differs drastically in the nonselfadjoint singular case. The basic spectral theory of nonselfadjoint singular second-order operators consisting of Sturm-Liouville theory was begun by Naimark, whose works initiated a deep study of spectral theory of nonselfadjoint operators $[3,4]$. He proved that the spectrum of a nonselfadjoint Sturm-Liouville operator consists of the continuous spectrum, the eigenvalues, and the spectral singularities. He also showed that these eigenvalues and spectral singularities are of finite number with finite multiplicities under certain conditions.

Later developments in this area concerned spectral analysis of the boundary value problems of the differential and discrete operators including Sturm-Liouville, Klein-Gordon, quadratic pencils of Schrödinger and Dirac-type operators within the context of determination of Jost solution and providing suffcient conditions guaranteeing the finiteness of the eigenvalues and spectral singularities [5-19]. 
In particular, boundary value problems including the integral boundary condition were first considered by Krall [20, 21]. He extended the work of Naimark [3] by applying a suitable integral boundary condition and generated the ordinary and nonhomogeneous expansion of a Sturm-Liouville operator.

Note that investigation of discrete analogues of ordinary differential operators is an important research area since difference equtions are well suited to find solutions with the aid of computers and can model many contemporary problems arising in control theory, biology, and engineering [7-11].

Let us denote by $l_{2}\left(\mathbb{N}, \mathbb{C}^{2}\right)$ the Hilbert space of all complex vector sequences $y=\left(\begin{array}{l}y_{n}^{(1)} \\ y_{n}^{(2)}\end{array}\right)_{n \in \mathbb{N}}$ with the inner product

$$
\langle y, u\rangle=\sum_{n \in \mathbb{N}}\left(y_{n}^{(1)} \overline{u_{n}^{(1)}}+y_{n}^{(2)} \overline{u_{n}^{(2)}}\right) .
$$

Consider the nonselfadjoint singular operator $L_{0}$ generated in the Hilbert space $l_{2}\left(\mathbb{N}, \mathbb{C}^{2}\right)$ by the discrete Dirac system

$$
\left\{\begin{array}{l}
y_{n+1}^{(2)}-y_{n}^{(2)}+p_{n} y_{n}^{(1)}=\lambda y_{n}^{(1)}, \\
-y_{n}^{(1)}+y_{n-1}^{(1)}+q_{n} y_{n}^{(2)}=\lambda y_{n}^{(2)},
\end{array} \quad n \in \mathbb{N},\right.
$$

and the boundary condition

$$
y_{0}^{(1)}=0,
$$

where $\lambda$ is a spectral parameter, $\Delta$ is the forward difference operator, and $p_{n}, q_{n} \in \mathbb{C}$. In [9] the integral representation for the Weyl function of $L_{0}$ and spectral expansion of the operator $L_{0}$ in terms of principal functions have been investigated in detail. Some generalization problems of the nonselfadjoint discrete Dirac operator have been subject to extensive studies in the literature. For instance, in [13] the general form of the operator $L_{0}$ has been considered for $n \in \mathbb{Z}$. Also, some authors investigated the problem with eigenparameterdependent boundary conditions $[10,14,15]$.

In this paper, we consider the operator $L$ generated in the Hilbert space $l_{2}\left(\mathbb{N}, \mathbb{C}^{2}\right)$ by the nonselfadjoint discrete Dirac equation (1.2) and boundary condition

$$
\sum_{n=0}^{\infty} h_{n} y_{n}=0
$$

where $\left(h_{n}\right)$ is complex vector sequence such that $h_{n}=\left(h_{n}^{(1)}, h_{n}^{(2)}\right), h_{n}^{(i)} \in l^{1}(\mathbb{N}) \cap l^{2}(\mathbb{N}), i=1,2$, $h_{0}^{(1)} \neq 0$. Clearly, $L_{0}$ is a particular case of $L$ for $h_{n}=(0,0), n \in \mathbb{N}=\{1,2, \ldots\}$. Differently from other studies, rather than considering an eigenparameter dependent boundary condition, we generalize the boundary condition (1.3) by using the orthogonality properties of $\left(y_{n}\right)$ with respect to vectors $\left(h_{n}\right)$. Therefore the conditions required for the finiteness of the eigenvalues and spectral singularities of the operator $L$ differ from the studies mentioned. Thus this paper presents the results in a more general and different approach.

The main objective of this paper is investigating the quantitative properties of the spectrum of the operator $L$. We apply and adopt the Naimark and Pavlov conditions on the 
potential and examine the eigenvalues and spectral singularities of the operator $L$ using the boundary uniqueness theorems of analytic functions.

Although the tools we use in this paper are basicly functional analysis techniques, the paper may lay the groundwork for future studies concerning the topics in direct and inverse problems, scattering theory, and applied physics.

The paper contains three sections. The first two are introductory, surveying all necessary results of the BVP (1.2)-(1.4). The last section focuses on the quantitative properties of the spectrum of the operator $L$.

\section{Jost solution of the operator $L$}

We will assume that

$$
\sum_{n=1}^{\infty} n\left(\left|p_{n}\right|+\left|q_{n}\right|\right)<\infty
$$

It is known from [9] that equation (1.2) has the solution

$$
\begin{aligned}
& f_{0}^{(1)}(z)=e^{i \frac{z}{2}}\left[1+\sum_{m=1}^{\infty} K_{0 m}^{11} e^{i m z}\right]-i \sum_{m=1}^{\infty} K_{0 m}^{12} e^{i m z}, \\
& f_{0}^{(2)}(z)=0
\end{aligned}
$$

and

$$
f_{n}(z)=\left(\begin{array}{l}
f_{n}^{(1)}(z) \\
f_{n}^{(2)}(z)
\end{array}\right)_{n \in \mathbb{N}}=\left\{\left[E_{2}+\sum_{m=1}^{\infty} K_{n m} e^{i m z}\right]\left(\begin{array}{c}
e^{i \frac{z}{2}} \\
-i
\end{array}\right) e^{i n z}\right\}, \quad n=1,2,3, \ldots
$$

for $\lambda=2 \sin \frac{z}{2}, E_{2}=\left(\begin{array}{ll}1 & 0 \\ 0 & 1\end{array}\right), K_{n m}=\left(\begin{array}{ll}K_{n m}^{11} & K_{n m}^{12} \\ K_{n m}^{21} & K_{n m}^{22}\end{array}\right), z \in \overline{\mathbb{C}}_{+}$. Note that the expressions $K_{n m}^{i j}, i, j=1,2$, can be written uniquely in terms of $\left\{p_{n}\right\}_{n \in \mathbb{N}}$ and $\left\{q_{n}\right\}_{n \in \mathbb{N}}$. Moreover, the inequality

$$
\left|K_{n m}^{i j}\right| \leq C \sum_{k=n+\left[\left|\frac{m}{2}\right|\right]}^{\infty}\left(\left|p_{k}\right|+\left|q_{k}\right|\right)
$$

is satisfied for $i, j=1,2$, where $\left[\left|\frac{m}{2}\right|\right]$ is the integer part of $\frac{m}{2}$, and $C>0$ is a constant. Hence $f_{n}(z)$ is analytic in $\mathbb{C}_{+}:=\{z \in \mathbb{C}: \operatorname{Im} z>0\}$ and continuous in $\overline{\mathbb{C}}_{+}:=\{z \in \mathbb{C}: \operatorname{Im} z \geq 0\}$. The function $f_{n}(z)$ is called the Jost solution of equation (1.2). Also, the following asymptotics hold [9]:

$$
\begin{aligned}
& \left(\begin{array}{l}
f_{n}^{(1)}(z) \\
f_{n}^{(2)}(z)
\end{array}\right)_{n \in \mathbb{N}}=\left[E_{2}+o(1)\right]\left(\begin{array}{c}
e^{i \frac{z}{2}} \\
-i
\end{array}\right) e^{i n z}, \quad z \in \overline{\mathbb{C}}_{+}, n \rightarrow \infty, \\
& \left(\begin{array}{l}
f_{n}^{(1)}(z) \\
f_{n}^{(2)}(z)
\end{array}\right)_{n \in \mathbb{N}}=\left[E_{2}+o(1)\right]\left(\begin{array}{c}
e^{i \frac{z}{2}} \\
-i
\end{array}\right) e^{i n z}, \quad n \in \mathbb{N}, z \in \overline{\mathbb{C}}_{+}, \operatorname{Im} z \rightarrow \infty .
\end{aligned}
$$

Let $\varphi_{n}(z)$ be a solution of (1.2) subject to the initial conditions

$$
\varphi_{0}^{(1)}(z)=0, \quad \varphi_{1}^{(2)}(z)=1,
$$


where

$$
\varphi_{n}(z)=\widetilde{\varphi}_{n}(\lambda)=\left\{\widetilde{\varphi}_{n}\left(2 \sin \frac{z}{2}\right)\right\}, \quad z \in \overline{\mathbb{C}}_{+}, n \in \mathbb{N} \cup\{0\} .
$$

Then $\varphi$ is an entire function, and

$$
\varphi(z)=\varphi(z+4 \pi) .
$$

The Wronskian of two solutions

$$
y_{n}=\left(\begin{array}{c}
c y_{n}^{(1)} \\
y_{n}^{(2)}
\end{array}\right)_{n \in \mathbb{N}}, \quad u_{n}=\left(\begin{array}{c}
c u_{n}^{(1)} \\
u_{n}^{(2)}
\end{array}\right)_{n \in \mathbb{N}}
$$

of (1.2) is defined by

$$
W[y, u]=y_{n}^{(1)} u_{n+1}^{(2)}-y_{n+1}^{(2)} u_{n}^{(1)} .
$$

Using the usual definition of Wronskian, we have

$$
W\left[f_{n}(z), \varphi_{n}(z)\right]=f_{0}^{(1)}(z), \quad z \in \overline{\mathbb{C}}_{+} .
$$

Let us define the semistrips $P_{0}:=\{z: z \in \mathbb{C},=x+i y, 0 \leq x<4 \pi, y>0\}$ and $P=P_{0} \cup[0,4 \pi)$.

Let us define

$$
N(z):=\sum_{n=0}^{\infty} h_{n} f_{n}(z)
$$

and also the functions,

$$
\begin{aligned}
& \widetilde{N}(z):=\sum_{n=0}^{\infty} h_{n} \varphi_{n}(z), \\
& \widehat{\varphi}_{n}(z):=\left(\varphi_{n}^{(1)}(z), \varphi_{n+1}^{(2)}(z)\right), \\
& \widehat{f}_{n}(z):=\left(f_{n}^{(1)}(z), f_{n+1}^{(2)}(z)\right), \\
& \widehat{\Omega}_{n}(z):=\left(\begin{array}{c}
\Omega_{n}^{(1)}(z) \\
\Omega_{n+1}^{(2)}(z)
\end{array}\right), \\
& S_{k}(z):=\frac{-1}{W[f, \varphi]}\left\{N(z) \widehat{\varphi}_{k}(z)+\widehat{N}(z) \widehat{f}_{k}(z)-\widehat{\varphi}_{k}(z) \sum_{n=k+1}^{\infty} h_{n} f_{n}(z)-\widehat{f}_{k}(z) \sum_{n=k+1}^{\infty} h_{n} \varphi_{n}(z)\right\} .
\end{aligned}
$$

For all $z \in P$ and $f_{0}^{(1)}(z) \neq 0$, the Green's function of the operator $L$ is obtained by standard techniques as

$$
G_{n k}(z)=G_{n k}^{(1)}(z)+G_{n k}^{(2)}(z),
$$

where

$$
G_{n k}^{(1)}(z)=\frac{S_{k}(z) f_{n}(z)}{N(z)},
$$


and

$$
G_{n k}^{(2)}(z)= \begin{cases}0, & k<n, \\ \frac{\widehat{f}_{k}(z) \varphi_{n}(z)+\widehat{\varphi}_{k}(z) f_{n}(z)}{f_{0}^{(1)}(z)}, & k \geq n .\end{cases}
$$

Obviously, for $\Omega=\Omega_{n}=\left(\begin{array}{c}\Omega_{n}^{(1)} \\ \Omega_{n}^{(2)}\end{array}\right) \in l^{2}\left(\mathbb{N}, \mathbb{C}^{2}\right)$,

$$
R_{\lambda}(L) \Omega_{n}:=\sum_{k=0}^{\infty} G_{n k}(z) \widehat{\Omega}_{k}, \quad n \in \mathbb{N} \cup\{0\},
$$

is the resolvent of the operator $L$.

It is also clear that $N(z)$ is the Jost function of the operator $L$ defined by using the Jost solution and boundary condition (1.4). The determination of Jost solutions plays an important role in spectral theory of discrete and differential operators. We refer the reader to books [1-4] for further details, which explain how this single function contains all the information about the spectrum of operators.

\section{Eigenvalues and spectral singularities of $L$}

Let us denote the set of eigenvalues and spectral singularities of the operator $L$ by $\sigma_{d}$ and $\sigma_{s s}$, respectively. From (2.5)-(2.7) and the definition of the eigenvalues and spectral singularities we have

$$
\begin{aligned}
\sigma_{d} & =\left\{\lambda: \lambda=2 \sin \frac{z}{2}, z \in P_{0}, N(z)=0\right\}, \\
\sigma_{s s} & =\left\{\lambda: \lambda=2 \sin \frac{z}{2}, z \in[0,4 \pi), N(z)=0\right\} .
\end{aligned}
$$

Let us define the sets

$$
\begin{aligned}
& M_{1}:=\left\{z: z \in P_{0}, N(z)=0\right\}, \\
& M_{2}:=\{z: z \in[0,4 \pi), N(z)=0\} .
\end{aligned}
$$

We also denote the set of all limit points of $M_{1}$ and $M_{2}$ by $M_{3}$ and $M_{4}$, respectively, and the set of all zeros in $P$ of $N(z)$ with infinite multiplicity by $M_{5}$. It then also follows that

$$
M_{1} \cap M_{5}=\emptyset, \quad M_{3} \subset M_{2}, \quad M_{4} \subset M_{2}, \quad M_{5} \subset M_{2},
$$

and the linear Lebesgue measures of $M_{2}, M_{3}, M_{4}$, and $M_{5}$ are zero. From the continuity of all derivatives of $N(z)$ on the real axis we have

$$
M_{3} \subset M_{5} \text { and } M_{4} \subset M_{5} \text {. }
$$

It is convenient to rewrite the sets of eigenvalues and spectral singularities of $L$ as

$$
\begin{aligned}
& \sigma_{d}=\left\{\lambda: \lambda=2 \sin \frac{z}{2}, z \in M_{1}\right\}, \\
& \sigma_{s s}=\left\{\lambda: \lambda=2 \sin \frac{z}{2}, z \in M_{2}\right\} .
\end{aligned}
$$


Theorem 3.1 Under conditions (2.1) and $h_{n}^{(i)} \in l^{1}(\mathbb{N}) \cap l^{2}(\mathbb{N}), i=1,2$, we have:

(i) The set of eigenvalues of $L$ is bounded and countable, and its limit points lie in [-2,2].

(ii) $\sigma_{s s} \subset[-2,2], \sigma_{s s}=\overline{\sigma_{s s}}$, and $\mu\left(\sigma_{s s}\right)=0$, where $\mu$ stands for the linear Lebesgue measure.

Proof From (2.3) and (2.4) we have the analyticity of $N(z)$ in the upper half-plane and the continuity of $N(z)$ on the real axis. For $\beta(z):=e^{-i \frac{z}{2}} N(z)$, we have the asymptotics

$$
\beta(z)=h_{0}^{(1)}+o(1), \quad \operatorname{Im} z>0, \operatorname{Im} z \rightarrow \infty
$$

Note that $\beta(z)$ and $N(z)$ have the same zeros except at infinity. Using (3.1), (3.2), and (3.4) and boundary uniqueness theorems of analytic functions [22], we arrive at (i) and (ii).

Definition 3.1 The multiplicity of a zero of $N(z)$ in the region $P$ is introduced as the multiplicity of the corresponding eigenvalue or spectral singularity of the operator $L$.

Now let us consider the condition

$$
\sum_{n=1}^{\infty} e^{\varepsilon n}\left(\left|p_{n}\right|+\left|q_{n}\right|+\left|h_{n}^{(i)}\right|\right)<\infty, \quad \varepsilon>0, i=1,2
$$

Theorem 3.2 Under condition (3.5), the operator L has a finite number of eigenvalues and spectral singularities, and each of them is of finite multiplicity.

Proof From (2.3) and (3.5) we observe that $N(z)$ has analytic continuation to the half-plane $\operatorname{Im} z>\frac{-\varepsilon}{2}$. Since $N(z)$ is a $4 \pi$-periodic function, the limit points of its zeros in $P$ cannot lie in $[0,4 \pi)$. Hence, using Theorem 3.1, we obtain the finiteness of eigenvalues and spectral singularities of $L$.

Note that condition (3.5), which is also known as Naimark's condition in the literature, ensures the analytic continuation of $N(z)$ from the real axis to the lower half-plane.

Now we will consider the Pavlov condition

$$
\sum_{n=1}^{\infty} e^{\varepsilon n^{\beta}}\left(\left|p_{n}\right|+\left|q_{n}\right|+\left|h_{n}^{(i)}\right|\right)<\infty, \quad \varepsilon>0, i=1,2, \frac{1}{2} \leq \beta<1,
$$

which is weaker than (3.5). Clearly, the function $N(z)$ is analytic in the upper half-plane and infinitely differentiable on the real axis. It is essential to notice at this point that $N(z)$ has no analytic continuation from the real axis to the lower half-plane. For this reason, we need to use a different method to investigate the finiteness of the eigenvalues and spectral singularities of $L$. We will benefit from the following lemma.

Lemma 3.3 ([9]) Suppose that the $4 \pi$-periodic function $\xi$ is analytic in the open halfplane, all of its derivatives are continuous in the closed upper half-plane, and

$$
\sup _{z \in P}\left|\xi^{(k)}(z)\right| \leq \eta_{k}, \quad k \in \mathbb{N} \cup\{0\}
$$


If the set $G$ with linear Lebesgue measure zero is the set of all zeros of the function $\xi$ with infinite multiplicity in $P$, and

$$
\int_{0}^{\omega} \ln t(s) d \mu\left(G_{s}\right)>-\infty
$$

where $t(s)=\inf _{k} \frac{\eta_{k} s^{k}}{k !}, k \in \mathbb{N} \cup\{0\}, \mu\left(G_{s}\right)$ is the Lebesgue measure of the s-neighborhood of $G$, and $\omega \in(0,4 \pi)$ is an arbitrary constant, then $\xi \equiv 0$.

Theorem 3.4 Assume that (3.6) holds. Then $M_{5}=\emptyset$.

Proof Under conditions (3.6), (2.2), (2.3), and (2.4), we obtain that

$$
\left|N^{(k)}(z)\right| \leq \eta_{k}, \quad k \in \mathbb{N} \cup\{0\}
$$

where

$$
\eta_{k}=2^{k} C \sum_{m=1}^{\infty} m^{k} \exp \left(-\varepsilon m^{\beta}\right)
$$

and $C>0$ is a constant. We have the following estimate:

$$
\eta_{k} \leq 2^{k} C \int_{0}^{\infty} x^{k} e^{-\varepsilon x^{\beta}} d x \leq D d^{k} k ! k^{k \frac{1-\beta}{\beta}},
$$

where $D$ and $d$ are constants depending $C$, $\varepsilon$, and $\beta$.

Applying the previous lemma to our case, we get that

$$
\int_{0}^{\omega} \ln t(s) d \mu\left(M_{5, s}\right)>-\infty
$$

where $t(s)=\inf _{k} \frac{\eta_{k} s^{k}}{k !}, k \in \mathbb{N} \cup\{0\}, \mu\left(M_{5, s}\right)$ is the Lebesgue measure of the $s$-neighborhood of $M_{5}$ and $\eta_{k}$ is defined by (3.8).

Now we have

$$
t(s) \leq D \exp \left\{-\frac{1-\beta}{\beta} e^{-1} d^{-\frac{\beta}{1-\beta}} s^{-\frac{\beta}{1-\beta}}\right\} .
$$

From (3.9) and (3.10) we get

$$
\int_{0}^{\omega} s^{-\frac{\beta}{1-\beta}} d \mu\left(M_{5, s}\right)>-\infty
$$

Since $\frac{1-\beta}{\beta} \geq 1,(3.11)$ holds for arbitrary s if and only if $\mu\left(M_{5, s}\right)=0$ or $M_{5}=\emptyset$.

Theorem 3.5 If condition (3.6) is satisfied, then the operator $L$ has a finite number of eigenvalues and spectral singularities, and each of them is of finite multiplicity.

Proof We have to show that the function $N(z)$ has a finite number of zeros with finite multiplicities in $P$. From (3.3) and the previous theorem we obtain that $M_{3}=M_{4}=\emptyset$. Hence 
the bounded sets $M_{1}$ and $M_{2}$ have no accumulation points, that is, $N(z)$ has only a finite number of zeros in $P$. Since $M_{5}=\emptyset$, these zeros are of finite multiplicity.

\section{Acknowledgements \\ Not applicable.}

Funding

Not applicable.

Availability of data and materials

Not applicable.

Competing interests

The authors declare that they have no competing interests.

Authors' contributions

Both authors contributed equally to each part of this work. Both authors read and approved the final manuscript.

\section{Publisher's Note}

Springer Nature remains neutral with regard to jurisdictional claims in published maps and institutional affiliations.

Received: 22 February 2020 Accepted: 22 July 2020 Published online: 06 August 2020

\section{References}

1. Levitan, B.M., Sargsian, I.S.: Introduction to Spectral Theory: Selfadjoint Ordinary Differential Operators, vol. 39. Am. Math. Soc., Providence (1975)

2. Chadan, K., Sabatier, P.C.: Inverse Problems in Quantum Scattering Theory. Springer, Berlin (2012)

3. Naimark, M.A.: Investigation of the spectrum and the expansion in eigenfunctions of a nonselfadjoint differential operator of the second order on a semi-axis. Transl. Am. Math. Soc. (2) 16,103-193 (1960)

4. Naimark, M.A.: Linear Differential Operators. Part II: Linear Differential Operators in Hilbert Space. Ungar, New York (1968) (Eng. Trans.)

5. Bairamov, E., Yokus, N.: Spectral singularities of Sturm-Liouville problems with eigenvalue-dependent boundary conditions. Abstr. Appl. Anal. 2009, 289596 (2009)

6. Adıvar, M., Bohner, M.: Spectral analysis of $q$-difference equations with spectral singularities. Math. Comput. Model. 43(7-8), 695-703 (2006)

7. Adıvar, M., Bairamov, E.: Spectral properties of non-selfadjoint difference operators. J. Math. Anal. Appl. 261(2), $461-478(2001)$

8. Yokus, N., Coskun, N.: Spectral properties of discrete Klein-Gordon s-wave equation with quadratic eigenparameter-dependent boundary condition. Iran. J. Sci. Technol. Trans. A, Sci. 43(4), 1951-1955 (2019)

9. Bairamov, E., Celebi, A.O.: Spectrum and spectral expansion for the non-selfadjoint discrete Dirac operators. Q. J. Math. 50(200), 371-384 (1999)

10. Bairamov, E., Koprubasi, T.: Eigenparameter dependent discrete Dirac equations with spectral singularities. Appl. Math. Comput. 215(12), 4216-4220 (2010)

11. Coskun, N., Yokus, N.: A note on the spectrum of discrete Klein-Gordon s-wave equation with eigenparameter dependent boundary condition. Filomat 33(2), 449-455 (2019)

12. Yokus, N., Coskun, N.: Spectral analysis of quantum Dirac systems. J. Nonlinear Sci. Appl. 10, 4524-4531 (2017)

13. Bairamov, E., Coskun, C.: The structure of the spectrum of a system of difference equations. Appl. Math. Lett. 18(4), 387-394 (2005)

14. Koprubasi, T., Mohapatra, R.N.: Spectral analysis of discrete Dirac equation with generalized eigenparameter in boundary condition. Filomat 33(18), 6039-6054 (2019)

15. Koprubasi, T.: Spectrum of the quadratic eigenparameter dependent discrete Dirac equations. Adv. Differ. Equ. 2014(1), 1 (2014)

16. Bairamov, E., Çakar, Ö., Krall, A.M.: Non-selfadjoint difference operators and Jacobi matrices with spectral singularities. Math. Nachr. 229(1), 5-14 (2001)

17. Bairamov, E., Karaman, O.: Spectral singularities of Klein-Gordon s-wave equations with an integral boundary condition. Acta Math. Hung. 97(1-2), 121-131 (2002)

18. Krall, A.M., Bairamov, E., Cakar, O.: Spectrum and spectral singularities of a quadratic pencil of a Schrödinger operator with a general boundary condition. J. Differ. Equ. 151(2), 252-267 (1999)

19. Yardimci, Ş., Arpat, E.K., Can, C.: On the structure of discrete spectrum of a non-selfadjoint system of differential equations with integral boundary condition. J. Math. Chem. 55(5), 1202-1212 (2017)

20. Krall, A.M.: A nonhomogeneous eigenfunction expansion. Trans. Am. Math. Soc. 117, 352-361 (1965)

21. Krall, A.M.: The adjoint of a differential operator with integral boundary conditions. Proc. Am. Math. Soc. 16(4), 738-742 (1965)

22. Dolzhenko, E.P.: Boundary-value uniqueness theorems for analytic functions. Math. Notes Acad. Sci. USSR 25(6), 437-442 (1979) 